\title{
Laser-Induced Crystalline Optical Waveguide on Glass Fibre
}

\author{
Xian Feng, Jindan Shi, Kevin Huang, Peh Siong Teh, Shaif-ul Alam, Morten Ibsen, Wei. H. Loh \\ Optoelectronic Research Centre, University of Southampton, SO17 1BJ, United Kingdom \\ xif@orc.soton.ac.uk
}

\begin{abstract}
We report for the first time the fabrication of a novel glass ribbon fibre with laser-induced single (or quasi-single) crystalline ( $\mathrm{La}, \mathrm{Yb}) \mathrm{BGeO}_{5}$ optical waveguide.
\end{abstract}

\section{Introduction}

Apart from the wide usage of low-loss glass fibre optical waveguide in the traditional telecom area [1], composite fibre optical waveguide devices, which are based on the combination of very different materials, such as glass/metal, glass/non-metallic dielectric crystal, glass/semiconductor, etc, have recently been suggested as one of the more promising routes for achieving various desired multiple functionalities, covering the areas of optics, electro-optics and magneto-optics [2].

Ferroelectric crystals are a class of materials capable of achieving multiple functionalities in optical waveguides. In particular, the spontaneous polarization of ferroelectric materials, arising from built-in electrical dipoles in the crystal structure, causes structural anisotropy and therefore results into nonlinear optical properties, such as the electro-optic effect, harmonic generation and photorefraction. Particularly the electro-optic effect has a fast response time of picoseconds or less, and is hence very useful for high-speed devices such as Mach-Zender interferometric switches [3].

One of the ferroelectric crystals, the stillwellite-type (La,Ln)BGeO ${ }_{5}$, in which $\mathrm{Ln}$ are group of 15 metallic chemical elements with atomic numbers 57 through 71 from lanthanum through lutetium - has attracted considerable attention, since continuous wave green laser emission due to self frequency doubling was observed in $\mathrm{Nd}^{3+}$ doped $\mathrm{LaBGeO}_{5}$ single crystals [4-6]. The second-order nonlinear coefficients $\left(d_{11}, d_{22}, d_{31}\right.$, and $\left.d_{33}\right)$ of undoped $\mathrm{LaBGeO}_{5}$ crystals were reported to be between $0.23-0.46 \mathrm{pm} / \mathrm{V}$ at $1.064 \mu \mathrm{m}$ [7], which is intermediate between the commonly used nonlinear crystal $\beta-\mathrm{BaB}_{2} \mathrm{O}_{4}$ (BBO) with absolute values of second-order nonlinear coefficient $\left|\mathrm{d}_{22}(1.064 \mu \mathrm{m})\right|=2.2 \mathrm{pm} / \mathrm{V}$ [7] and poled Gedoped $\mathrm{SiO}_{2}$ fibre with $\mathrm{d}_{33}$ of $0.054 \mathrm{pm} / \mathrm{V}$ [8]. $\mathrm{LaBGeO}_{5}$ crystals also have attractive ferroelectric properties such as pyroelectricity. Furthermore, $\mathrm{LaBGeO}_{5}$ melt can be easily vitrified to a glass solid with the same composition (i.e., $50 \mathrm{GeO}_{2}-25 \mathrm{~B}_{2} \mathrm{O}_{3}-25(\mathrm{La}, \mathrm{Ln})_{2} \mathrm{O}_{3}$, mol.\%, or LBGO) without rapid quenching [9], because the large quantity of strong bonding glass former oxides $\left(\mathrm{GeO}_{2}\right.$ and $\left.\mathrm{B}_{2} \mathrm{O}_{3}\right)$ effectively suppresses the tendency towards crystallization. Laser induced single-crystalline architectures with observed second harmonic generation were previously reported in $\mathrm{Sm}_{0.5} \mathrm{La}_{0.5} \mathrm{BGeO}_{5}$ glass bulk [10]. In laser induced crystallization, the glass absorbs the laser power as heat and gets melted within very local areas. Then crystallization occurs when the melt cools down to solid with certain cooling rate.

In this work, we choose a novel ribbon optical fibre based on LBGO glass as the host. Using UV $244 \mathrm{~nm}$ laser, (La, Yb) $\mathrm{BGeO}_{5}$ crystalline waveguides have been fashioned on the optical fibre. No obvious grain boundary is observed along the crystalline waveguide. This is the first report of using laser-inducing method to fabricate crystalline waveguide in optical fibre.

\section{Fabrication of glass fibre and surface channel waveguide}

The LBGO glass was prepared by a conventional melting-quenching method. Stoichiometric amounts of $\quad \mathrm{Yb}_{2} \mathrm{O}_{3}, \quad \mathrm{La}_{2} \mathrm{O}_{3}$ (99.99\%), $\mathrm{B}_{2} \mathrm{O}_{3}(99.99 \%)$ and $\mathrm{GeO}_{2}(99.99 \%)$ were weighed in the composition of $50 \mathrm{GeO}_{2}$ $25 \mathrm{~B}_{2} \mathrm{O}_{3}-17.5 \mathrm{La}_{2} \mathrm{O}_{3}-7.5 \mathrm{Yb}_{2} \mathrm{O}_{3}$ (mol.\%) to provide a 70-gram batch, and melted in a platinum crucible at $14500^{\circ} \mathrm{C}$ for 90 minutes. The melt was then casted into a stainless steel mould, which was preheated at $600^{\circ} \mathrm{C}$, to form a rectangular slab preform with dimensions of $5 \times 15 \times 75 \mathrm{~mm}$. The glass slab was then annealed around glass transition temperature $\mathrm{T}_{\mathrm{g}}$ for 2hours. The annealed slab preform was drawn in ribbon shape fibre with a width of $450 \mu \mathrm{m}$ and a thickness of $150 \mu \mathrm{m}$. The yield of the fibre draw was $>50$ meters.

A UV 244nm CW laser was employed to write channel waveguides on the surface of the ribbon fibre. As schematically described in Fig.1, The fibre was fixed on a house-made grooved metal plate with wax and then mounted on a programmable, motor-controlled high-resolution $X Y Z$ translation stage. The collimated UV laser beam was focused on the surface of the ribbon 
through a focal lens. The focused spot size was $\sim 15 \mu \mathrm{m}$ in diameter. $5-15 \mathrm{~cm}$ long channel waveguides were successfully fabricated on the top surface of the fibre ribbons.

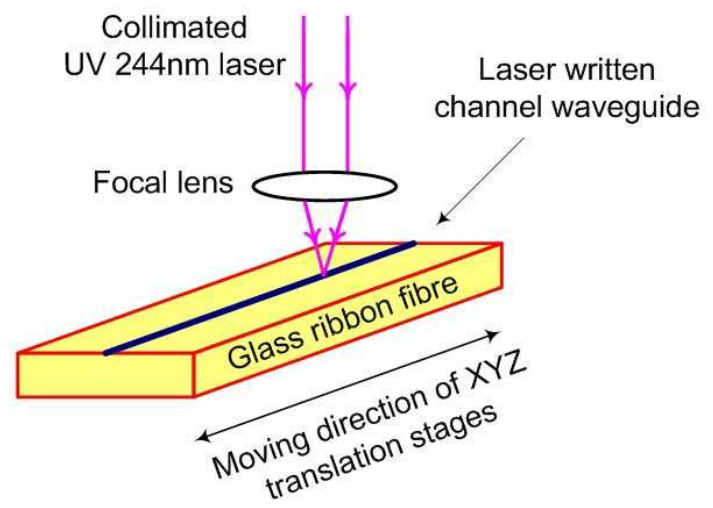

Fig. 1: Schematic of fabricating laser induced crystalline waveguide on glass ribbon fibre

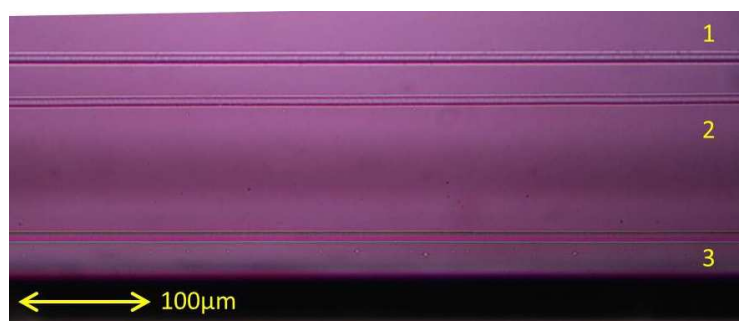

Fig. 2: Optical photograph of top view of the channel waveguides written by UV laser

Characterization of the crystalline waveguide Fig.2 shows the optical microscope picture of the top view of the induced waveguides on one fibre sample. The used laser power and the moving speed of the stage along the fibre were set as $20 \mathrm{~mW}$ and $60 \mathrm{~mm}$ per minute, respectively. The width of the channel was $\sim 5.0 \mu \mathrm{m}$ under such conditions. Mind that it took only 2.5 minutes to write such a channel with the length of $15 \mathrm{~cm}$.

The spontaneous Raman scattering spectra of the glass bulk and the LBGO glass fibre were measured with a Renishaw Raman Microscope using a depolarized $632.8 \mathrm{~nm} \mathrm{HeNe}$ laser. The focused spot size on the sample is $5 \mu \mathrm{m}$ in diameter. As shown in Fig.3, the Raman spectra of the glass slab preform and the ribbon fibre without UV laser processing are virtually identical, with highly amorphous features. On the other hand, the Raman spectrum inside the UV-induced channel waveguide (the channel 1 in Fig.2) shows multiple narrow sharp lines, indicating a well grown crystalline phase (or phases). From the literature [11], this crystalline phase is similar to the standard undoped $\mathrm{LaBGeO}_{5}$ crystal. But the additional peaks at $750 \mathrm{~cm}^{-1}, 1100 \mathrm{~cm}^{-1}$, and $1200-1500 \mathrm{~cm}^{-1}$ are believed to be due to the lower symmetry of the crystal structure of the heavily $\mathrm{Yb}$ doped ( $\mathrm{La}$,
$\mathrm{Yb}) \mathrm{BGeO}_{5}$, in comparison with $\mathrm{LaBGeO}_{5}$. In crystal, the large difference of the ion radius between $\mathrm{La}^{3+}$ and $\mathrm{Yb}^{3+}(103$ and $86.8 \mathrm{pm}$ respectively [12]) results into a large change of the crystal structure, particularly on the symmetry and the related bonds of the forming units $\left[\mathrm{GeO}_{4}\right]$ and $\left[\mathrm{BO}_{4}\right]$.

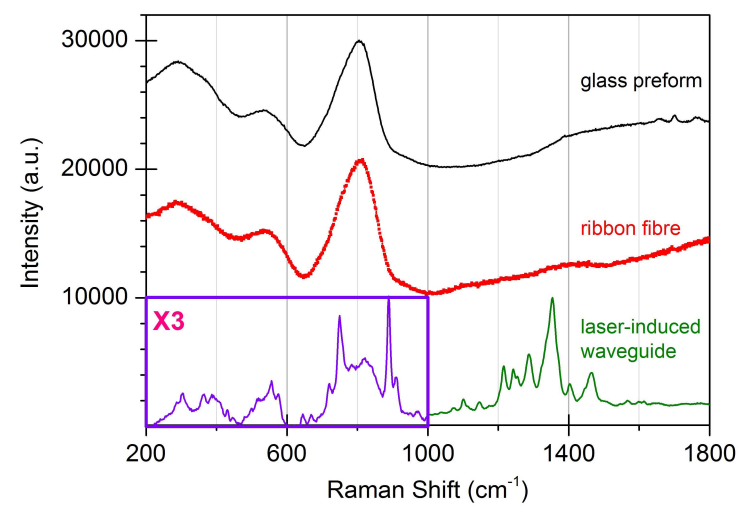

Fig. 3: Raman spectrum of glass slab preform, ribbon fibre, and laser induced waveguide. Note that within the Raman shift from 200 to $1000 \mathrm{~cm}^{-1}$, the intensity of the Raman spectrum (inset) of the waveguide was multiplied by a factor of 3 for a clearer representation.

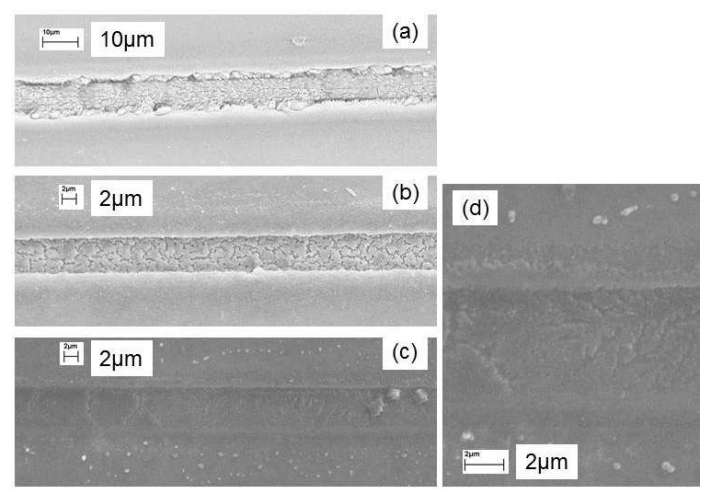

Fig. 4: SEM photographs of channel waveguides with different writing conditions. In (a), (b) and (c), the laser power was 53, 38, and $20 \mathrm{~mW}$, and the scanning speed is 20,60 , and $60 \mathrm{~mm} / \mathrm{min}$, respectively. (d): zoom-in view of (c).

Fig.4 shows the scanning electron microscopy (SEM) photograph of the top view of the laser-induced crystalline channels on ribbon fibre with various exposure laser powers and scanning speeds. It is seen that by reducing the laser power and increasing the scanning speed, the grain boundaries within the crystalline channel almost vanish from (a) to (c). Note that the waveguide in Fig.4(c) is the same channel 1 shown in Fig.2. Fig.4(d) is the zoom-in view of Fig.4(c), showing that there are some features with dimension of $\sim 200 \mathrm{~nm}$ on the surface of the waveguide. These may be the residual grain boundaries or just the surface submicron cracks in the crystalline waveguide. Based on the above characterization, therefore, we infer that 
single crystalline or quasi single crystalline waveguide has fabricated through UV-induced crystallization approach.

It is believed that within the local area exposed to the UV laser beam, the glass got remelt and with certain cooling rate, polycrystalline grains or single crystal can be formed. But in principle, by optimizing the laser power, scanning speed, and the laser wavelength for varying the laser penetrate depth, one of the initial grains can act as the seed for growing single crystal so that single crystalline waveguide with controllable cross-section geometry and dimensions along the laser scanning direction can be made.

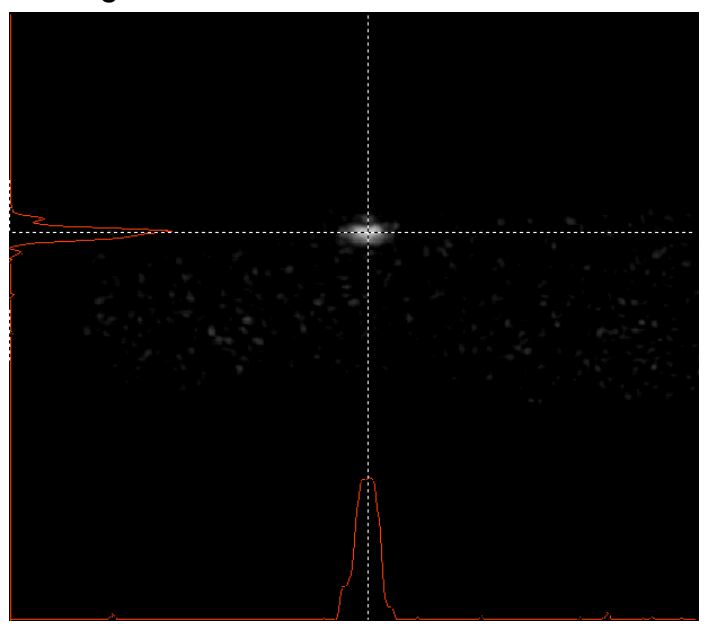

Fig. 5: near field image of guidance at 1060nm

Fig.5 shows the near field image of the guidance at $1060 \mathrm{~nm}$ from the $4.7 \mathrm{~cm}$ long channel 1 in Fig.2. It is seen that at least four lower-order modes are guided in the core. According to the refractive index $n$ of the LBGO glass (1.80 at $1.06 \mu \mathrm{m}$ [9]), the absolute index increase of the crystalized core from the glass cladding is estimated to be 0.01 or higher, i.e., with a NA $>0.15$. This is consistent with the reported index difference between the $\mathrm{LaBGeO}_{5}$ surface crystallization layer and glass, 0.016 at 632.8nm [9]. From scattered light measurement, the loss of the crystalline waveguide is estimated to be $3 \mathrm{~dB} / \mathrm{cm}$, which includes the strong absorption tail of $\mathrm{Yb}^{3+}$ at $1060 \mathrm{~nm}$ due to the extremely high doping level ( 19wt.\%).

By optimizing the fabrication procedure, single mode performance and low background loss should be expected in this laser-induced crystalline waveguide.

\section{Perspective of crystalline waveguide on glass fibre}

Although improvements on the optical performance of the laser-induced crystalline waveguide on fibre need to be done before this novel waveguide can be practical, such waveguides can be very promising as electrooptical medium, nonlinear optical medium, active laser medium, etc. The surface channel waveguide can also be used as a sensor device like the conventional D-shaped fibre. In addition, the laser power and writing speed in the previous reported laser-induced single crystalline structures in the LBGO glass bulk were $\sim 1 \mathrm{~W}$ and $<0.1 \mathrm{~mm} / \mathrm{min}$, respectively [10]. The $\mathrm{mW}$-level exposed laser power and fast writing speed $(>5 \mathrm{~cm} / \mathrm{min})$ used in this work makes the fabrication show that our approach is practical and efficient.

\section{Conclusions}

In summary, we present for the first time a laser induced single or quasi-single crystalline channel waveguide on a glass ribbon fibre. This novel fibre optical waveguide based on composite materials holds promise for multiple functionalities.

\section{Acknowledgements}

This work is supported by UK Engineering and Physical Sciences Research Council, under the EPSRC Centre for Innovative Manufacturing in Photonics.

\section{References}

[1] K.C.Kao et al., Proc. IEE 113, 1151 (1966).

[2] P.Sazio et al., Science, 311, 1583 (2006).

[3] H. Jain, Ferroelectrics, 306, 111 (2004).

[4] A.A.Kaminskii et al., Phys. Status. Solidi (a) 125, 671 (1991).

[5] J.Capmany et al., Appl. Phys. Lett. 70, 2517 (1997).

[6] J.Capmany et al., Appl. Phys. Lett. 72, 531 (1998).

[7] D.N.Nikogosyan, Nonlinear Optical Crystals: A Complete Survey, Springer (2005).

[8] A.Canagasabey et al., Opt. Lett. 34, 2483 (2009).

[9] Y.Takahashi et al., J. Appl. Phys. 89, 5282 (2001).

[10] P.Gupta et al., J. Am. Ceram. Society, 91, 110, (2008).

[11] Y.Takahashi et al., J. Ceram. Society Jpn. 116, 1108 (2008).

[12] N.N.Greenwood et al., Chemistry of the Elements, Butterworth-Heinemann (1997). 